\title{
An overview of cervical cancer epidemiology and prevention in Bulgaria
}

\author{
Milena Karcheva, ${ }^{1, *}$, Angel Yordanov ${ }^{2}$, Sergey Kostadinov ${ }^{3}$
}

Abstract

Introduction Cervical cancer ranks as the fourth leading cause of female cancer in Bulgaria. The objective of the study was to describe the current burden of cervical cancer in Bulgaria for a one-year period and prevention options.

Methods In this retrospective epidemiological study, online database from National Statistical Institute-Sofia, National Center of Public Health and Analyses-Sofia, National Center of Infectious and Parasitic Diseases-Sofia for one year were analyzed. Annual data were presented in numbers and per 100 000 of population.

Results A total of 15,759 women in Bulgaria were diagnosed with cervical cancer in 2018. Morbidity is 23.5 of 100000 population. Mortality of malignancy of the cervix in 2018 per 100000 populations was 8.4 among women. Differences of mortality have been recorded as per statistical regions. A low immunization range has been recorded of vaccine against human papilloma virus (HPV) - 7.2\% (girls 13 years of age) and $12.8 \%$ (girls 12 years of age) for first dose and $11.9 \%$ (girls 12 year of age) for second dose in 2019.

Conclusions High morbidity and mortality from cervical cancer were registered in Bulgaria. The immunization coverage was low. The strategy for disease prevention through mandatory screening and mandatory immunizations needs to be rethought.

Keywords Incidence, mortality, HPV, vaccine coverage.

\section{Introduction}

According to the World Health Organization (WHO), cervical cancer is the fourth most frequent cancer in women with an estimated total 570000 new cases in 2018 representing $6.6 \%$ of all female cancers. Approximately $90 \%$ of deaths from cervical cancer occurred in lowand middle-income countries.

Received: 09 June 2020; revised: 02 August 2020; accepted: 17 August 2020.

${ }^{1} \mathrm{MD}, \mathrm{PhD}$, Assoc. Prof., Department of Epidemiology, Faculty of Public Health, Medical University-Pleven, Pleven, 5800, Bulgaria; ${ }^{2} \mathrm{MD}$, PhD, Chief Assis. Prof., Department of Midwifery, Faculty of Health Care, Medical UniversityPleven, Pleven, 5800, Bulgaria; ${ }^{3} \mathrm{MD}$, PhD, Chief Assis. Prof., Department of General medicine, Forensic Medicine and Deontology, Medical University-Pleven, Pleven, 5800, Bulgaria.

${ }^{*}$ Corresponding author: Milena Karcheva, milena karcheva@abv.bg

Article downloaded from www.germs.ro

Published December 2020

(c) GERMS 2020

ISSN $2248-2997$

ISSN - L = $2248-2997$
Women at risk for cervical cancer are: the female population aged $\geq 15$ years. Other factors contributing to cervical cancer were tobacco smoking, oral contraceptive use among women, immune status - people who are immune compromised, such as those living with human immunodeficiency virus (HIV), are more likely to have persistent HPV infections and a more rapid progression to pre-cancer and cancer; burden of cervical HPV infection and other HPV-related cancers, co-infection with other sexually transmitted agents, sexual behavior, percentage of 15-year-olds who have had sexual intercourse (men/women), middle-aged at first sexual intercourse (men/women), cervical cancer screening coverage. Performed HPV vaccination reduces the risk of getting of cervical cancer. ${ }^{1.5}$

The role of papilloma viruses in the oncogenesis of cervical cancer has been described by Prof. Dr. Harald zur Hausen. He identified HPV16 and HPV18 in cervical cancers in 19831984. Vaccination against HPV has been available since 2006. There are currently three vaccines protecting against both HPV 16 and 18, which are known to cause at least $70 \%$ of cervical 
cancers. WHO considers the three vaccines equally protective against cervical cancer. HPV vaccines work best if administered prior to exposure to HPV. ${ }^{6}$

Therefore, WHO recommends vaccinating girls, aged between 9 and 14 years, when most have not started sexual activity. Some countries have started to vaccinate boys as the vaccination prevents genital cancers in males. HPV vaccination does not replace cervical cancer screening. In countries where HPV vaccine is introduced, screening programs may still need to be developed or strengthened.

Republic of Bulgaria is a country in Southeastern Europe. The population of Bulgaria is 7360000 people according to the 2011 national census. Population as of 31 December 2018 - in total 7000 039. Women - 3604338 (50\%). The majority of the population, $72.5 \%$, reside in urban areas. A serious problem for women's health in the country is cervical cancer. $^{7,8}$

The objective of the study was to describe the current burden of cervical cancer in Bulgaria for a one-year period and the options of prevention.

\section{Methods}

\section{Data source}

Online data available from the National Statistical Institute-Sofia, National Center of Public Health and Analyses-Sofia for 2018 were analyzed. Data for immunization were available from the National Center of Infectious and Parasitic Diseases-Sofia for 2018 and 2019 years.

\section{Data description}

Annual data are presented in number of cases and number per 100000 of population (incidence, mortality). Data are presented by sex, 5-year age groups, type of the settlement and cause of death at national level and by statistical regions and districts in accordance with the Nomenclature of territorial units for statistics in Bulgaria. In statistics of healthcare in Bulgaria is applied the International classification of the disease (ICD), X-th Revision. Bulgaria is divided into six statistical regions: Northwestern, Northcentral, Northeastern, Southwestern, Southcentral and Southeastern.

\section{Results}

A total of 15759 women in Bulgaria were diagnosed with cervical cancer in 2018. Among them, the new cases were 850 , with a morbidity of 23.5 of 100000 population. The women under dispensary surveillance and control were 14882 (94.43\%) - Table 1.

Table 1. Cervical cancer mortality (per 100000 of population) by statistical regions in Bulgaria, 2018

\begin{tabular}{|l|c|}
\hline Region & Female \\
\hline 1. Northwestern & 15.4 \\
\hline 2. Northcentral & 7.9 \\
\hline 3. Northeastern & 9.4 \\
\hline 4. Southwestern & 6.7 \\
\hline 5. Southcentral & 7.0 \\
\hline 6. Southeastern & 8.3 \\
\hline
\end{tabular}

The mortality of cervix malignancy in 2018 per 100000 population of Bulgaria was 8.4 among women. Information for mortality from cervical cancer by regions is presented in Figure 1.

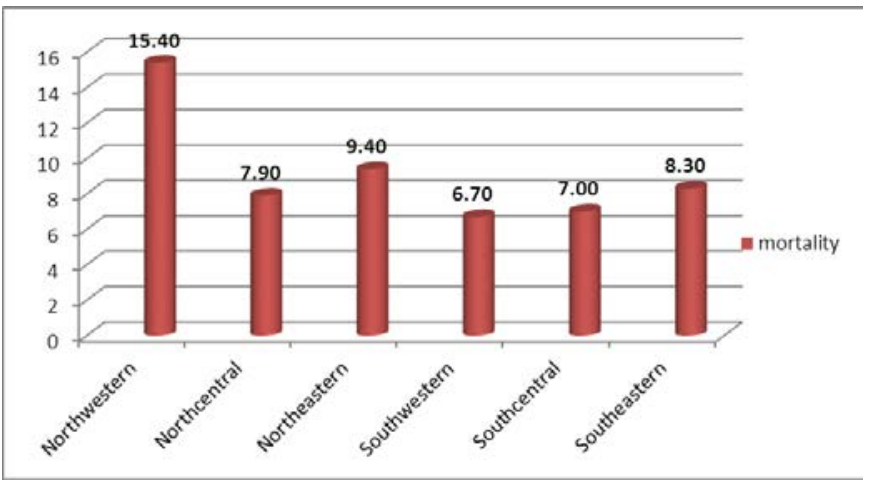

Figure 1. Statistical regions in Bulgaria and mortality of cervical carcinoma in the regions

The highest mortality is recorded in the Northwest statistical area that includes the following districts: Vidin, Montana, Vratsa, Lovech, Pleven. The reported mortality from cervical cancer in them is presented in Figure 2. 


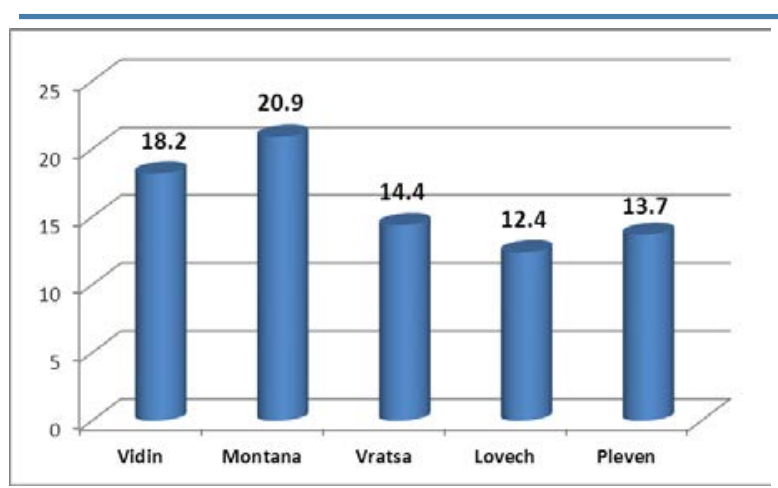

Figure 2. Cervical carcinoma mortality by district in the Northwestern region in Bulgaria (2018)

The North central statistical region includes following districts: Gabrovo, Veliko Tarnovo, Ruse, Razgrad, Silistra. The reported mortality from cervical cancer in them is presented in Figure 3.

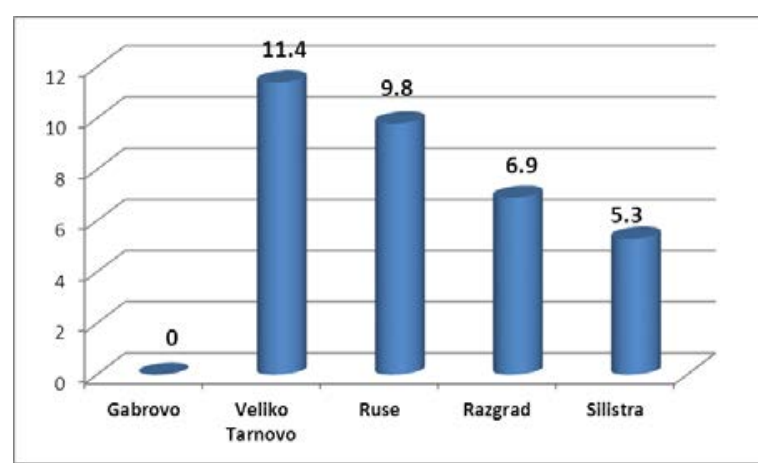

Figure 3. Cervical carcinoma mortality by district in the Northcentral region in Bulgaria (2018)

The Northeastern statistical region includes the following districts: Dobrich, Varna, Shumen, Targovishte. The reported mortality from cervical cancer in them is presented in Figure 4.

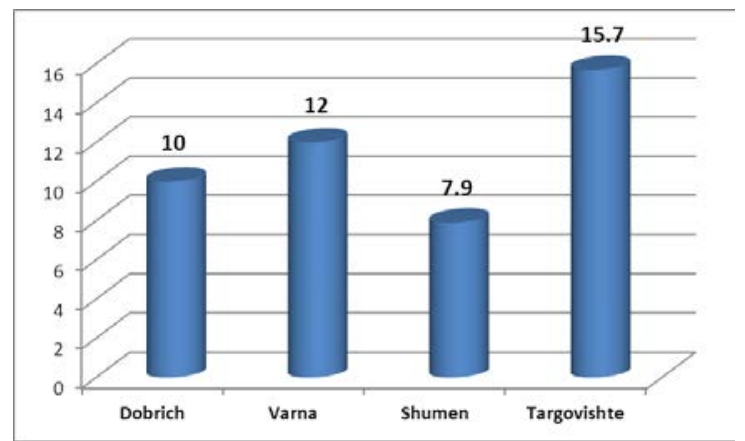

Figure 4. Cervical carcinoma mortality by district in the Northeastern region in Bulgaria (2018)
The Southwestern statistical region includes the following districts: Blagoevgrad, Kyustendil, Pernik, Sofia, Sofia capital. The reported mortality from cervical cancer in them is presented in Figure 5. The lowest mortality rate was recorded in that region of Bulgaria.

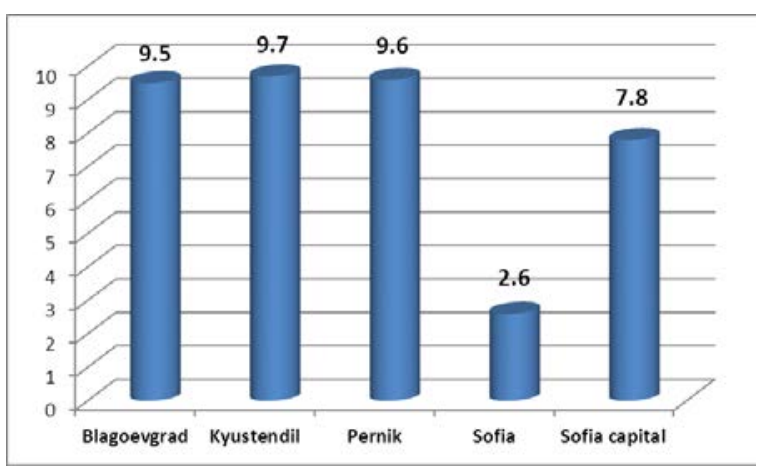

Figure 5. Cervical carcinoma mortality by district in the Southwestern region in Bulgaria (2018)

The South central statistical region includes the following districts: Kardzhali, Pazardzhik, Plovdiv, Smolyan, Haskovo. The reported mortality from cervical cancer in them is presented in Figure 6.

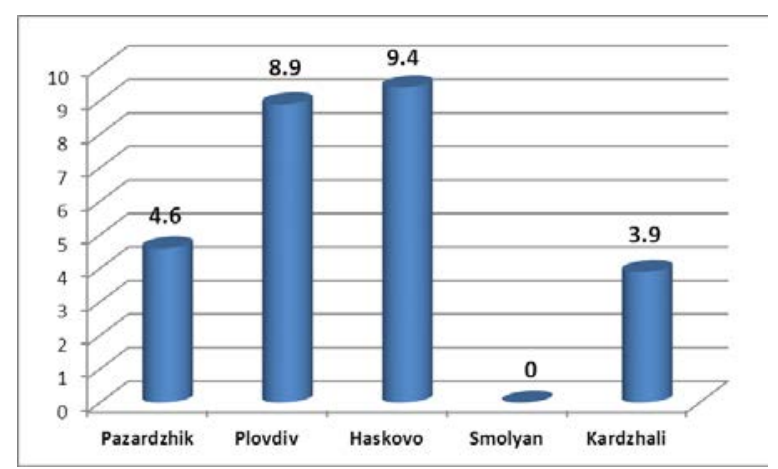

Figure 6. Cervical carcinoma mortality by district in the Southcentral region in Bulgaria (2018)

The Southeastern statistical region includes the following districts: Burgas, Sliven, Stara Zagora, Yambol. The reported mortality from cervical cancer in them is presented in Figure 7. 


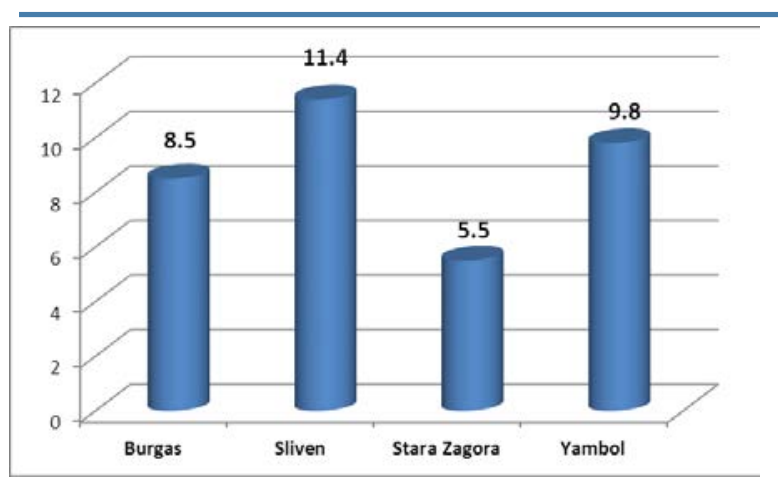

Figure 7. Cervical carcinoma mortality by district in the Southeastern region in Bulgaria (2018)

According to Regulations № 15 for immunizations in Republic of Bulgaria and in compliance with the National program for primary prophylactics of cervical cancer (20172020), dated 2012, immunizations against HPV are introduced at girls, 12 and 13 years old. ${ }^{9,10}$ The immunization scheme consists of two dozes.

The information for conducted immunizations in 2018-2019 are shown in Table 2. A low immunization range has been recorded of vaccine against human papilloma virus (HPV) - 7.2\% (girls 13 year of age) and $12.8 \%$ (girls 12 year of age) for first dose and $11.9 \%$ (girls 12 year of age) for the second dose in 2019. For 2018, the official data were incomplete. Immunized against HPV with the complete regimen ( 3 doses above 14 years) for the other age groups - 44 persons in 2018 and 77 in 2019.

\section{Discussion}

High incidence and unfavorable forecast of cervical cancer define its significance as considerable problem of modern healthcare. That malignancy is the fourth most encountered disease among women in Bulgaria. Our survey established that the incidence in Bulgaria is higher than the average in Europe, thus taking one of the first places in the European Union. ${ }^{8}$

According to the European Cancer Information System (ECIS) the estimated incidence by country shows that the age standardized rates (European New) per 100000 were between 5.0 and 32.2. Countries were classified in five groups: 1 ) with highest morbidity between 26.8 and 32.2 are Lithuania, Estonia,
Romania and Bulgaria, that is countries from Eastern Europe (expected incidence for Bulgaria is 28.1); 2) with incidence between 21.3 and 26.8 are Latvia, Hungary and Slovakia; 3) with incidence between 15.9 and 21.3 is Czech Republic; 4) with incidence between 10.4 and 15.4 are Poland, Ireland, Portugal, Greece, Germany, Belgium, Sweden, Norway, Denmark, Croatia; 5) with lowest incidence between 5.0 and 10.4 are France, Finland, United Kingdom, Italy, Iceland, Austria, Cyprus, Luxemburg, Slovenia, Malta, The Netherlands, Switzerland. ${ }^{11}$

Regarding mortality of cervical cancer, ECIS estimated mortality provided the following information: 1) with highest mortality between 3.4 and 16.3 in Europe is Romania; 2) estimated mortality between 10.6 and 13.4 are Bulgaria (11.9), Lithuania and Latvia; 3) estimated mortality between 7.7 and 10.6 was estimated in Estonia; 4) mortality between 4.9 and 7.7 was estimated in Portugal, Ireland, Croatia; 5) lowest mortality between 2.0 and 4.9 was estimated in all other countries. ${ }^{11}$

Our data show that different values of cervical cancer are registered in different regions of Bulgaria. The cancer burden was highest in the northern half of the country. Most probably that is in connection to the social-economic status of northern Bulgaria. The northern-western region is the poorest and undeveloped for decades, that impacts on the health status of the population. A similar statement is made by other of our authors for recent period, who associate the lower incidence in south Bulgaria with religion, noting lower intensity among Muslim women, actually the manner of life. ${ }^{2}$

During the recent years, steps have been undertaken to overcome that unfavorable trend. In 2012 the „National program for primary prophylactics of cervical cancer in $\mathrm{R}$ of Bulgaria 2012-2016" was adopted, continuing for the time 2017-2020. In the same year immunizations with HPV vaccines were introduced for girls, completed 12 and 13 years. ${ }^{10,11}$ In order to achieve optimum effect of the implementation of vaccines, it is required to maintain high immunization range, which in our country is not to be observed. The low immunization range has its subjective and objective reasons. On one part 
Table 2. Immunization against human papillomavirus in Bulgaria (2018/2019)

\begin{tabular}{ccccccc}
\hline $\begin{array}{c}\text { Immunization } \\
\text { scheme }\end{array}$ & \multicolumn{3}{c}{$\begin{array}{c}\text { First dose } \\
\text { 2018/2019 }\end{array}$} & \multicolumn{3}{c}{$\begin{array}{c}\text { Second dose } \\
2018 / 2019\end{array}$} \\
\hline $\begin{array}{c}\text { Age of } \\
\text { immunization }\end{array}$ & Subject, & Immunized, & Immunization & Subject, & Immunized, & Immunization \\
Number & Number & coverage, $\%$ & number & number & coverage, $\%$ \\
12 years & $-/ 22402$ & $3075 / 2875$ & $-/ 12.8$ & $-/ 19123$ & $2315 / 2273$ & $-/ 11.9$ \\
13 years & $-/ 19325$ & $1535 / 1397$ & $-/ 7.2$ & $-/$ & $1070 / 436$ & $\%$ \\
\hline
\end{tabular}

*These numbers represent the two different years; the available data is incomplete.

the vaccine has the perspective to reduce morbidity and on the other part in the population there is a lack of sufficient motivation for vaccination; that imposes a more comprehensive approach to prophylactics measures as a whole. Similar results are seen in other countries of Eastern Europe, e.g., Romania that records high morbidity and mortality, low immunization coverage, influence from antivaccine movements. ${ }^{12}$

Cervical cancer is one of the leading in frequency and mortality oncological disease among women, at the same time one of the most easily preventive and permitting early diagnostics. For that purpose, it is required to implement in the country mandatory immunization, as well as mandatory screening for women. We think that solving the issue of the mass screening and early detection of cervical cancer shall result to reduce the incidence of the cervical carcinoma. Indicative in that regard is the prospective investigation of 25 years period in UK, where they established that at regular screening of women, the mortality rate of cervical cancer could be prevented by $80 \% .{ }^{13}$

Nowadays, the activities on prophylactics of general practitioner doctors include: Pap smear for women between 30 and 40 years, in whom after two consecutive negative results, examination is performed every 3 years. Those activities of the National Health Insurance Fund (NHIF) are not sufficient of Bulgarian women in view of the high morbidity and mortality. It is required to expand the age range of women to be tested.

An efficient campaign is also required to increase the level of awareness of women regarding the importance of screening as well as health knowledge of youngsters on early detection and prevention of sexual transmitted diseases and through them of cancer. In recent years there were carried out studies of health culture of the population, attitudes and competencies, which gives reason to think that work is in the right direction. ${ }^{14}$

\section{Conclusions}

High morbidity and mortality from cervical cancer were registered in Bulgaria. The immunization coverage was low. The strategy for disease prevention through mandatory screening and mandatory immunizations needs to be rethought.

Authors' contributions statement: MK carried out the conception and designing of the study and wrote the manuscript. AY supervised the data collection, assisted with interpretation of the data and reviewed the manuscript. SK assisted with interpretation of the data and reviewed the manuscript. All authors read and approved the final version of the manuscript.

Conflicts of interest: All authors - none to declare.

Funding: None to declare.

References

1. Crosbie EJ, Einstein MH, Franceschi S, Kitchener HC. Human papillomavirus and cervical cancer. Lancet 2013;382:889-99.

https://doi.org/10.1016/S0140-6736(13)60022-7

2. Samson KK, Haynatzki G, Soliman AS, Valerianova Z. Temporal changes in the cervical cancer burden in Bulgaria: Implications for eastern european countries going through transition. Cancer Epidemiol 2016;44:154-60. https://doi.org/10.1016/j.canep.2016.08.014

3. Bruni L, Albero G, Serrano B, et al. ICO/IARC Information Centre on HPV and Cancer (HPV Information Centre). Human papillomavirus and 
related diseases in Bulgaria. Summary Report 17 June 2019. Accessed on: 17 June 2019. Available at: https://hpvcentre.net/statistics/reports/BGR.pdf

4. Momenimovahed Z, Salehiniya H. Incidence, mortality and risk factors of cervical cancer in the world. BRAT 2017;4:1795-811.

https://doi.org/10.15419/bmrat.v4i12.386

5. Kovachev SM. Cervical cancer and vaginal microbiota changes. Arch Microbiol 2020;202:323-7. https://doi.org/10.1007/s00203-019-01747-4

6. World Health Organization. Immunization, Vaccines and Biologicals. Accessed on: 01 May 2017. Available at:

https://www.who.int/immunization/diseases/hpv/en/

7. National Statistical Institute. Sofia. Accessed on: 01 December $2019 \quad$ Available at: https://www.nsi.bg/en/content/5623/mortality-causessex-statistical-regions-and-districts

8. Ministry of Health. National Center of Public Health and Analyses. Sofia. Accessed on: 01 December 2019. Available at: https://ncpha.government.bg/bg/
9. Ministry of Health. Regulation no. 15 on immunizations in the Republic of Bulgaria [in Bulgarian].

10. National Program for Primary Prevention of Cervical Cancer (2017-2020) [in Bulgarian].

11. National Center of Infectious and Parasitic Diseases. Sofia. Accessed on: 01 January 2020. Available at: https://www.ncipd.org/index.php?option=com k2\&vi ew=item\&id=190:aboutncipd\&Itemid=1191\&lang=en

12. ECIS - European Cancer Information System Accessed on: 01 January 2020. Available at: https://ecis.jrc.ec.europa.eu/explorer.php

13. Landy R, Pesola F, Castañón A, Sasieni P. Impact of cervical screening on cervical cancer mortality: estimation using stage-specific results from a nested case-control study. Br J Cancer 2016;115:1140-6. https://doi.org/10.1038/bjc.2016.290

14. Irmov V. Contemporary problems related to cervical cancer prevention. Dissertation. Varna, 2018 [in Bulgarian].

Please cite this article as:

Karcheva M, Yordanov A, Kostadinov S. An overview of cervical cancer epidemiology and prevention in Bulgaria. GERMS. 2020;10(4):322-327. doi: 10.18683/germs.2020.1224 